\title{
Renormalization scale setting for D-meson mixing
}

\author{
Alexander Lenz $\odot,{ }^{1,2, *}$ Maria Laura Piscopo $\odot,{ }^{1, \dagger}$ and Christos Vlahos ${ }^{1, \hbar}$ \\ ${ }^{1} I P P P$, Department of Physics, University of Durham, DH1 3LE Durham, United Kingdom \\ ${ }^{2}$ Physik Department, Universität Siegen, Walter-Flex-Straße 3, 57068 Siegen, Germany
}

(Received 20 July 2020; accepted 7 October 2020; published 13 November 2020)

\begin{abstract}
A naive application of the heavy quark expansion (HQE) yields theory estimates for the decay rate of neutral $D$ mesons that are 4 orders of magnitude below the experimental determination. It is well known that this huge suppression results from severe Glashow-Iliopoulos-Maiani cancellations. We find that this mismatch can be solved by individually choosing the renormalization scale of the different internal quark contributions. For $b$ and $c$ hadron lifetimes, as well as for the decay rate difference of neutral $B$ mesons, the effect of our scale setting procedure lies within the previously quoted theory uncertainties, while we get enlarged theory uncertainties for the semileptonic $C P$ asymmetries in the $B$ system.
\end{abstract}

DOI: 10.1103/PhysRevD.102.093002

\section{INTRODUCTION}

An improvement of our theoretical understanding of charm physics is crucial to make use of the huge amount of current and future experimental charm data obtained by LHCb [1], BESIII [2], and Belle II [3]. The recent discovery of direct $C P$ violation in the charm system by the LHCb Collaboration [4] is an example of this necessity. Briefly after the announcement of a nonvanishing measurement of $\Delta A_{C P}=A_{C P}\left(D^{0} \rightarrow K^{+} K^{-}\right)-A_{C P}\left(D^{0} \rightarrow \pi^{+} \pi^{-}\right)$, both theory papers arguing for a beyond standard model [5,6] (partly based on the calculation of Ref. [7]) and a standard model (SM) [8-11] origin of this measurement appeared (a summary of references investigating a previous claim for evidence of $C P$ violation can be found in Ref. [12]). Thus, a decisive conclusion about the potential size of the SM contribution to $\Delta A_{C P}$ is mandatory to fully exploit the significant experimental progress in this field. A long-standing puzzle in this regard is the theoretical description of mixing of neutral $D$ mesons. Charm mixing is by now experimentally well established and the HFLAV [13] average of Refs. [4,14-46] (in the case of allowed $C P$ violation) reads

$x=\frac{\Delta M_{D}}{\Gamma_{D^{0}}}=(0.37 \pm 0.12) \%, \quad y=\frac{\Delta \Gamma_{D}}{2 \Gamma_{D^{0}}}=0.68_{-0.07}^{+0.06 \%}$,

\footnotetext{
*alexander.josef.lenz@gmail.com

maria.1.piscopo@durham.ac.uk

christos.vlahos@durham.ac.uk
}

Published by the American Physical Society under the terms of the Creative Commons Attribution 4.0 International license. Further distribution of this work must maintain attribution to the author(s) and the published article's title, journal citation, and DOI. Funded by SCOAP. where $\Delta M_{D}$ is the mass difference of the two mass eigenstates of the neutral $D^{0}$ mesons and $\Delta \Gamma_{D}$ the corresponding decay rate difference. However, theory predictions for $x$ and $y$ cover a vast range of values - differing by several orders of magnitude, see e.g., the compilations of theory predictions in Refs. $[47,48]$. Future measurements will not only increase the precision of $x$ and $y$, but also give stronger bounds or even a measurement of the $C P$ violation in mixing [49] encoded e.g., in the phase $\phi_{12}$, see Eq. (3), which is currently constrained to be within $\left[-2.5^{\circ}, 1.8^{\circ}\right]$ [13]. A reliable range of potential SM values is pivotal to benefit from the coming experimental improvements.

\section{HQE}

The heavy quark expansion (HQE) [50-56] (see Ref. [57] for a recent overview) describes the total decay rate of heavy hadrons and the decay rate difference of heavy neutral mesons as an expansion in inverse powers of the heavy quark mass. In the case of $B_{s}$-mixing and $b$ hadron lifetimes, the HQE predicts values [57-63] which are in good agreement with the experimental ones [13].

\begin{tabular}{lll}
\hline & HFLAV 2019 & HQE 2019 \\
\hline$\frac{\tau\left(B_{s}\right)}{\tau\left(B_{d}\right)}$ & $0.994(4)$ & $1.0007(25)$ \\
$\frac{\tau\left(B^{+}\right)}{\tau\left(B_{d}\right)}$ & $1.076(4)$ & $1.082_{-0.026}^{+0.022}$ \\
$\frac{\tau\left(\Lambda_{b}\right)}{\tau\left(B_{d}\right)}$ & $0.969(6)$ & $0.935(54)$ \\
$\Delta \Gamma_{B_{s}}$ & $0.091(13) \mathrm{ps}^{-1}$ & $0.090(5) \mathrm{ps}^{-1}$ \\
\hline
\end{tabular}

This impressive result when the expansion parameter is $\Lambda / m_{b}$ ( $\Lambda$ denotes an hadronic scale of the order of $\left.\Lambda^{\mathrm{QCD}}\right)$ suggests that one might still get reasonably well-behaving estimates moving to the charm system, where the expansion parameter increases by a factor of 3 . For the lifetime ratio $\tau\left(D^{+}\right) / \tau\left(D^{0}\right)$, both NLO-QCD corrections to the 
dimension-six contribution [64] and values for the nonperturbative matrix elements of four quark operators [58] are known-for all other charm hadrons, this is not yet the case; thus, corresponding theory estimates have to be taken with care - and one finds indeed a nice agreement within the huge theory uncertainties,

$$
\left.\frac{\tau\left(D^{+}\right)}{\tau\left(D^{0}\right)}\right|^{\text {HFLAV } 2019}=2.536(19),\left.\quad \frac{\tau\left(D^{+}\right)}{\tau\left(D^{0}\right)}\right|^{\text {HQE } 2019}=2.7_{-0.8}^{+0.7}
$$

Hence, it is quite surprising that a naive application of the $\mathrm{HQE}$ fails completely for $D$-mixing.

\section{CHARM MIXING}

Diagonalizing the two-dimensional mixing matrix of the $D^{0}$ and the $\bar{D}^{0}$ meson-containing the off-diagonal matrix elements $M_{12}$ and $\Gamma_{12}$-one gets

$x_{12}=\frac{2\left|M_{12}\right|}{\Gamma_{D^{0}}}, \quad y_{12}=\frac{\left|\Gamma_{12}\right|}{\Gamma_{D^{0}}}, \quad \phi_{12}=\arg \left(\frac{M_{12}}{\Gamma_{12}}\right)$,

while $x$ and $y$ depend on both $M_{12}$ and $\Gamma_{12}$. The calculation of $M_{12}$ is beyond the scope of the present work; hence, we can only determine one contribution to the mixing phase $\phi_{12}$, also for $\Delta \Gamma_{D}$ we will use the bound $\Delta \Gamma_{D} \leq 2\left|\Gamma_{12}\right|$ (see e.g., Refs. $[65,66])$. Within the HQE, $\Gamma_{12}$ is expanded as

$$
\Gamma_{12}=\left[\Gamma_{3}^{(0)}+\frac{\alpha_{s}}{4 \pi} \Gamma_{3}^{(1)}+\ldots\right] \frac{\left\langle Q_{6}\right\rangle}{m_{c}^{3}}+\ldots,
$$

where the ellipsis stands for terms of higher order. Equation (4) is diagrammatically represented in Fig. 1. The product of $\Delta C=1$ operators in the effective

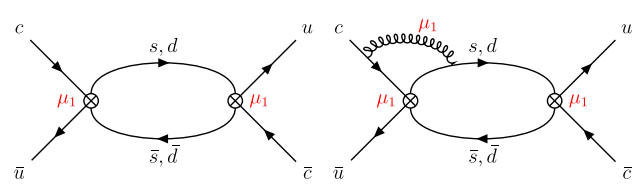

(a)

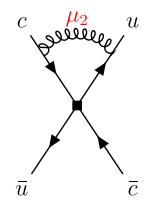

(b)
FIG. 1. (a) Diagrams describing mixing of neutral $D$ mesons via intermediate $s \bar{s}, s \bar{d}, d \bar{s}$, and $d \bar{d}$ states in the full theory at LOQCD (left) and NLO-QCD (right). The crossed circles denote the insertion of $\Delta C=1$ operators of the effective Hamiltonian describing the charm-quark decay. The dependence on the renormalization scale $\mu_{1}$ in the Wilson coefficients cancels against the $\mu_{1}$ dependence of the QCD corrections. (b) Diagram describing mixing of neutral $D$ mesons at NLO-QCD in the HQE. The full dot indicates the insertion of $\Delta C=2$ operators. The dependence on the renormalization scale $\mu_{2}$ cancels between the QCD corrections to the diagram and the matrix element of the corresponding operators.
Hamiltonian ("full" theory) is matched into a series of local $\Delta C=2$ operators $Q_{n}$ of increasing dimension $n \geq 6$, with the short distance coefficients denoted by $\Gamma_{n-3}^{(i)}$. The expressions for $\Gamma_{3}^{(i)}$ can be simply obtained from the corresponding ones for $B$-mixing given in Refs. [67-72] while the matrix elements of the dimension-six operators have been determined in e.g., Refs. [58,73]. Experiments yield a small value for the decay rate difference

$$
\Delta \Gamma_{D}^{\mathrm{Exp}}=2 y / \tau\left(D^{0}\right)=(0.032 \pm 0.003) \mathrm{ps}^{-1},
$$

which leads to the following bound:

$$
\Delta \Gamma_{D}^{\mathrm{Exp}} \geq 0.028 \mathrm{ps}^{-1}
$$

at 1 standard deviation. Below we will investigate the quantities

$$
\alpha=-\arg \left(\Gamma_{12}\right), \quad \Omega=\frac{2\left|\Gamma_{12}\right| \mathrm{SM}}{0.028 \mathrm{ps}^{-1}},
$$

where $\alpha$ contributes to $C P$ violation in mixing and values of $\Omega$ smaller than 1 indicate a failure of our theoretical framework to describe $D$-mixing within the 1 sigma range. A naive application of the HQE leads to $\Omega=3.4 \times 10^{-5}$ at leading order (LO)-QCD $\left(6.2 \times 10^{-5}\right.$ at next-to-leading order (NLO)-QCD), i.e., the HQE prediction of the decay rate difference is more than 4 orders of magnitude smaller than the experimental determination. Correspondingly, the phase $\alpha$ is very large, i.e., $\alpha=93^{\circ}$ at $\mathrm{LO}-\mathrm{QCD}\left(\alpha=99^{\circ}\right.$ at NLO-QCD). By default, in our numerical analysis, we use Particle Data Group [74] values for the quark ( $\overline{\mathrm{MS}})$ and meson masses as well as for the strong coupling, CabibboKobayashi-Maskawa (CKM) elements from Ref. [75], nonperturbative matrix elements from Ref. [58], and the $D^{0}$ decay constant from Ref. [76].

\section{GIM IN D-MIXING}

In order to better understand the peculiarities of D-mixing, we decompose $\Gamma_{12}$ according to the flavor of the internal quark pair. The three contributions are denoted $\Gamma_{12}^{s s}, \Gamma_{12}^{d d}$, and $\Gamma_{12}^{s d}$,

$$
\begin{aligned}
\Gamma_{12}= & -\left(\lambda_{s}^{2} \Gamma_{12}^{s s}+2 \lambda_{s} \lambda_{d} \Gamma_{12}^{s d}+\lambda_{d}^{2} \Gamma_{12}^{d d}\right) \\
= & -\lambda_{s}^{2}\left(\Gamma_{12}^{s s}-2 \Gamma_{12}^{s d}+\Gamma_{12}^{d d}\right) \\
& +2 \lambda_{s} \lambda_{b}\left(\Gamma_{12}^{s d}-\Gamma_{12}^{d d}\right)-\lambda_{b}^{2} \Gamma_{12}^{d d} .
\end{aligned}
$$

$\lambda_{q}=V_{c q} V_{u q}^{*}$ is the CKM element and we have used the unitarity relation $\lambda_{d}+\lambda_{s}+\lambda_{b}=0$ to eliminate $\lambda_{d}$. Equation (8) shows very pronounced hierarchies,

$$
-\lambda_{s}^{2}=-4.791 \times 10^{-2}+3.094 \times 10^{-6} I,
$$




$$
\begin{gathered}
+2 \lambda_{s} \lambda_{b}=+2.751 \times 10^{-5}+6.121 \times 10^{-5} I, \\
-\lambda_{b}^{2}=+1.560 \times 10^{-8}-1.757 \times 10^{-8} I .
\end{gathered}
$$

The CKM factor in the first term of Eq. (8) has by far the largest real part, while the second term has actually the largest imaginary part-it should thus be important for the determination of the potential size of $C P$ violation in mixing. Since the relative imaginary part of $\lambda_{b}$ is much larger than that of $\lambda_{s}$, we suggest to keep all terms in Eq. (8). Furthermore, extreme Glashow-Iliopoulos-Maiani (GIM) cancellations [77] affect the coefficients of the CKM elements in Eq. (8). Expanding in the small mass parameter $z=m_{s}^{2} / m_{c}^{2}$, we find at LO-QCD (top line) and at NLOQCD (lower line),

$$
\begin{gathered}
\Gamma_{12}^{s s}=\left\{\begin{array}{l}
1.62-2.34 z-5.07 z^{2}+\ldots, \\
1.42-4.30 z-12.45 z^{2}+\ldots,
\end{array}\right. \\
\Gamma_{12}^{s d}-\Gamma_{12}^{d d}=\left\{\begin{array}{l}
-1.17 z-2.53 z^{2}+\ldots, \\
-2.15 z-6.26 z^{2}+\ldots,
\end{array}\right. \\
\Gamma_{12}^{s s}-2 \Gamma_{12}^{s d}+\Gamma_{12}^{d d}=\left\{\begin{array}{l}
-13.38 z^{3}+\ldots, \\
0.07 z^{2}-29.72 z^{3}+\ldots
\end{array}\right.
\end{gathered}
$$

It was observed before [78,79] that QCD corrections lower the GIM suppression by one power of $z$. The peculiarity of Eq. (8) is that the CKM dominant factor $\lambda_{s}^{2}$ multiplies the extremely GIM suppressed term given in Eq. (14), the CKM suppressed factor $\lambda_{s} \lambda_{b}$ multiplies the GIM suppressed term given in Eq. (13), and the very CKM suppressed factor $\lambda_{b}^{2}$ multiplies $\Gamma_{12}^{d d}$, where no GIM suppression is present. Thus, the three contributions in Eq. (8) have actually a similar size,

$$
\begin{aligned}
\Gamma_{12}= & \left(2.08 \times 10^{-7}-1.34 \times 10^{-11} I\right)(\text { first term }) \\
& -\left(3.74 \times 10^{-7}+8.31 \times 10^{-7} I\right)(\text { second term }) \\
& +\left(2.22 \times 10^{-8}-2.5 \times 10^{-8} I\right)(\text { third term }) .
\end{aligned}
$$

It is also clear that a sizeable phase in $D$-mixing can only arise, if the slightly GIM suppressed term is enhanced. Different solutions have been suggested in order to explain the mismatch between the HQE prediction and experimental determination.

(i) Higher orders in the HQE could be less affected by the GIM suppression [80-82] — first estimates of the dimension nine contribution [83] to $D$-mixing show indeed such an enhancement, but not on a scale to reproduce the experimental number. For a final conclusion about this possibility, a full determination of dimension nine and twelve would be necessary.

(ii) Quark hadron duality violations as large as the deviation between experiments and the naive HQE prediction-yielding $\Omega \approx 10^{-5}$ —seem unlikely given the many successful tests of the HQE as stated above. In fact, in Ref. [66], it was shown that violations as small as 20 percent could be sufficient to explain the experimental value of $D$-mixing.

(iii) The HQE is not applicable and we have to rely on different methods, like summing over the exclusive decays channels contributing to the decay rate difference; see e.g., Refs. [84-86].

\section{ALTERNATIVE SCALE SETTING}

In $\Gamma_{12}$, the two renormalization scales $\mu_{1}$ and $\mu_{2}$ arise; see Fig. 1. $\mu_{1}$ is the scale at which the $\Delta C=1$ Wilson coefficients of the effective Hamiltonian and the QCD corrections in the full theory are computed and up to higher order terms, this scale dependence has to cancel between the two sources, Fig. 1(a). Similarly, radiative corrections to the HQE diagrams introduce the dependence on the scale $\mu_{2}$, Fig. 1(b), the latter cancels against the scale dependence of the matrix element of the corresponding $\Delta C=2$ operators. We will not discuss the $\mu_{2}$-dependence any further since this cancellation is very effective. For the $\mu_{1}$-dependence, in the $B_{s}$ system, the cancellation is numerically only weakly realized when moving from LO-QCD to NLO-QCD, see Refs. [87,88]. This indicates the importance of higher order corrections and first steps in that direction show indeed large NNLO-QCD effects $[87,88]$. In the $D$ system a reduction of the $\mu_{1}$-dependence, when moving from LO-QCD to NLO-QCD, is present in the individual contributions $\Gamma_{12}^{s s, s d, d d}$ but not in $\Gamma_{12}$, see Fig. 2, which seems to be again a consequence of the severe GIM cancellations. Making the scale dependence explicit, we can write

$$
\Gamma_{12}=\sum_{q_{1} q_{2}=s s, s d, d d} \Gamma_{3}^{q_{1} q_{2}}\left(\mu_{1}^{q_{1} q_{2}}, \mu_{2}^{q_{1} q_{2}}\right)\left\langle Q_{6}\right\rangle\left(\mu_{2}^{q_{1} q_{2}}\right) \frac{1}{m_{c}^{3}}+\ldots
$$

In general, different internal quark pairs contribute to different decay channels of the $D^{0}\left(\bar{D}^{0}\right)$ meson e.g., $s \bar{s}$ to

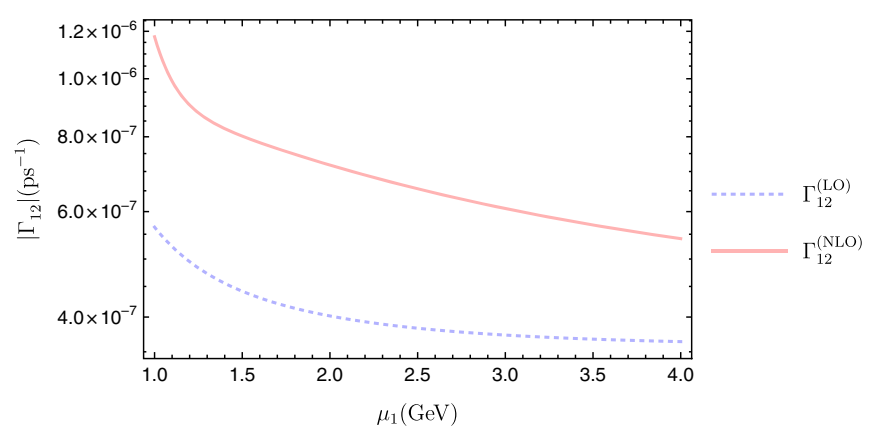

FIG. 2. Comparison of $\mu_{1}$-dependence of $\left|\Gamma_{12}\right|$ at LO-QCD (dotted purple) and NLO-QCD (solid pink). 
a $K^{+} K^{-}$final state and $s \bar{d}$ to a $\pi^{+} K^{-}$final state. For each of these different observables, the choice of the renormalisation scales is a priori arbitrary; nevertheless, one typically fixes $\mu_{x}^{s s}=\mu_{x}^{s d}=\mu_{x}^{d d}=\mu$ which is then chosen to be equal to the mass of the decaying heavy quark, i.e., $\mu=m_{Q}$ for $Q$ quark decays, to minimize terms of the form $\alpha_{s}(\mu) \ln \left(\mu^{2} / m_{Q}^{2}\right)$. Uncertainties due to unknown higher order corrections are estimated varying $\mu$ between $m_{Q} / 2$ and $2 m_{Q}$-in the case of the charm quark, we fix the lower bound to $1 \mathrm{GeV}$ in order to still ensure reliable perturbative results.

Here we propose two different ways to treat the renormalization scale $\mu_{1}^{q_{1} q_{2}}$, both will reduce the mismatch between the HQE prediction and the experimental determination of $D$-mixing, while leaving the other HQE predictions unchanged:

(i) $\mu_{1}^{s s}, \mu_{1}^{s d}$, and $\mu_{1}^{d d}$ are set to the common scale $m_{c}$ but varied independently between $1 \mathrm{GeV}$ and $2 m_{c}$.

(ii) $\mu_{1}^{s s}, \mu_{1}^{s d}$, and $\mu_{1}^{d d}$ are set to different scales according to the size of the available phase space. In particular, we will evaluate $\Gamma_{3}^{s s}$ at the scale $\mu_{1}^{s s}=\mu-2 \epsilon, \Gamma_{3}^{s d}$ at the scale $\mu_{1}^{s d}=\mu-\epsilon$, and $\Gamma_{3}^{d d}$ at the scale $\mu_{1}^{d d}=\mu$, where $\epsilon$ is related to the kinematics of the decays.

If $\epsilon$ is not too large, then both methods will yield results for the individual $\Gamma_{3}^{s s}, \Gamma_{3}^{s d}$, and $\Gamma_{3}^{d d}$ which lie within the usually quoted theory uncertainties obtained following the prescription stated above, but they will clearly affect in a sizable way the severe GIM cancellations in Eqs. (13) and (14). The first method gives a considerably enhanced range of values for $\Omega$,

$$
\Omega \in\left[4.6 \times 10^{-5}, 1.3\right],
$$

which nicely covers also the experimental determination of the decay rate difference. Scanning independently over $\mu_{1}^{s s}$, $\mu_{1}^{s d}$, and $\mu_{1}^{d d}$ in 11 equidistant steps, we find that out of the 1331 points only 14 give a value of $\Omega<0.001$, while 984 give a value of $\Omega>0.1$. The very small HQE prediction seems thus to be an artifact of fixing the scales $\mu_{1}^{s s}, \mu_{1}^{s d}$, and $\mu_{1}^{d d}$ to be the same. The range of values shown in Eq. (17) is similar even if we use the pole scheme for the quark masses, lattice results instead of the HQET results, or a different $\Delta C=2$ operator basis. In all these cases, the value $\Omega \geq 1$ can be obtained. For $\alpha$, we get in general results ranging from $-\pi$ to $\pi$. A closer look, however, shows that for $\Omega>0.5$ only values of $\alpha<0.1^{\circ}$ are allowed, while large values of $\alpha$ can only be obtained if the theory prediction for $y$ is inconsistent with the experimental determination. The second method for the scale setting requires the introduction of a mass scale $\epsilon$. A possible estimate for the size of this parameter could be the strange quark mass $\epsilon=m_{s} \approx 0.1 \mathrm{GeV}$ or the phase space difference of the corresponding exclusive decay channels: comparing the energy release of $D^{0} \rightarrow K^{+} K^{-}$, $M_{D^{0}}-2 M_{K^{+}}=0.88 \mathrm{GeV}$, with that of $D^{0} \rightarrow \pi^{+} \pi^{-}$,

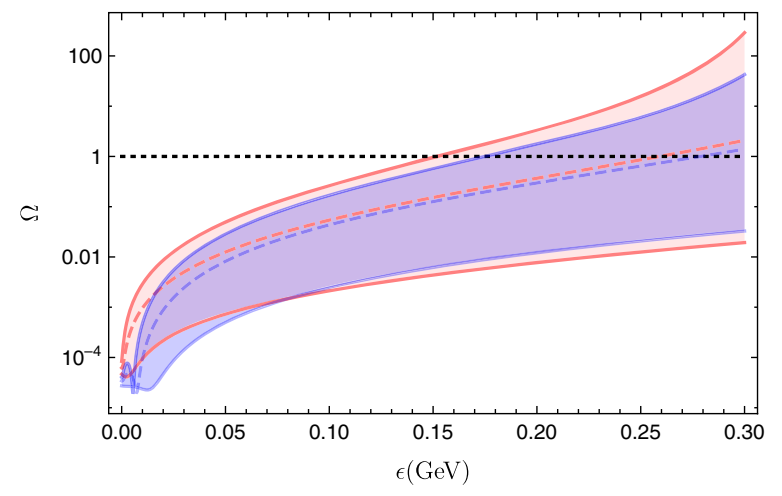

FIG. 3. Comparison of the $\epsilon$ dependence of $\Omega$ at LO-QCD (purple) and NLO-QCD (pink) for different values of $\mu$ : the dashed line corresponds to $\mu=m_{c}$ while the two solid lines to $\mu=1 \mathrm{GeV}$ and $\mu=2 m_{c}$.

$M_{D^{0}}-2 M_{\pi^{+}}=1.59 \mathrm{GeV}$, we might expect that $\epsilon \approx$ $0.35 \mathrm{GeV}$. Figure 3 shows how the HQE prediction of $\Omega$ would be affected in this scenario. Again, an enhancement up to the experimental value is possible for values of $\epsilon \approx 0.2 \mathrm{GeV}$. Finally, we have to test the effect of our alternative scale setting procedure on all the other $\mathrm{HQE}$ predictions. For the lifetimes (i.e., $\tau\left(D^{+}\right) / \tau\left(D^{0}\right)$ as well as $b$ hadron lifetimes) and the decay rate difference $\Delta \Gamma_{s}$, no GIM-like cancellations arise and we can only get a shift within the usually quoted theory range. But the semileptonic $C P$ asymmetries are governed by the weakly GIM suppressed contribution in $B_{s}$-mixing. Within the SM, we get

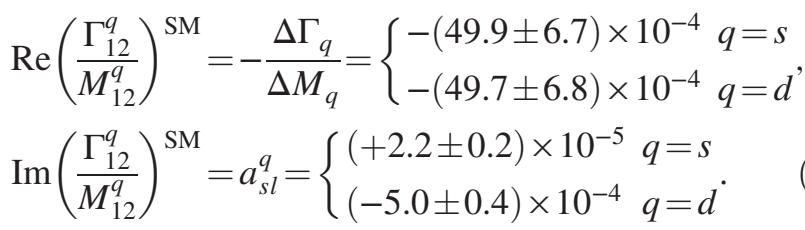

Performing the $\epsilon$ analysis, we obtain

\begin{tabular}{|c|c|c|}
\hline$\epsilon(\mathrm{GeV})$ & $\Gamma_{12}^{s} / M_{12}^{s}$ & $\Gamma_{12}^{d} / M_{12}^{d}$ \\
\hline 0 . & $-0.00499+0.000022 I$ & $-0.00497-0.00050 I$ \\
\hline 0.2 . & $-0.00494+0.000023 I$ & $-0.00492-0.00053 I$ \\
\hline 0.5 . & $-\mathbf{0 . 0 0 4 8 4}+0.000026 I$ & $-\mathbf{0 . 0 0 4 8 2}-0.00059 I$ \\
\hline 1.0 & $-\mathbf{0 . 0 0 4 4 7}+0.000037 I$ & $-\mathbf{0 . 0 0 4 4 8}-0.00084 I$ \\
\hline 1.5 . & $-0.00287+0.000091 I$ & $-0.00309-0.0021 I$ \\
\hline
\end{tabular}

We see that for values of $\epsilon$ up to $1 \mathrm{GeV}$ the predictions for the real part lie with the usually quoted theory uncertainties (indicated in bold). The predictions for the semileptonic asymmetries can, however, be increased by almost $100 \%$ compared to the usually quoted values.

\section{CONCLUSIONS}

Our main finding is that the range of the HQE uncertainty for $y$ is much larger than previously thought, and it 
covers the experimental value if we modify the usually adopted scale setting. For a full solution of the $D$-mixing puzzle, we nevertheless suggest a more precise estimate of higher order corrections in the HQE, as well as a completion of the NNLO-QCD corrections to the leading term.

The alternative scale setting procedure shows that a small contribution to $C P$ violation in mixing stemming from the decay rate can be up to one per mille within in the SM, which agrees with estimates made in Refs. [89,90]. For a prediction of $C P$ violation in mixing, the contribution coming from $M_{12}$ has to be determined in addition. This might be done in future via the help of dispersion relations, see e.g., Refs. [85,90,91]. We would like to note that our suggested procedure still respects the GIM mechanism, because for vanishing internal strange quark mass, also the parameter $\epsilon$ will be zero.
Finally, this scale setting does not affect quantities like $\tau\left(D^{+}\right) / \tau\left(D^{0}\right), b$ hadron lifetimes, and $\Delta \Gamma_{s}$ outside the range of their quoted theoretical errors, but it affects the semileptonic $C P$ asymmetries and we get enhanced SM ranges,

$a_{s l}^{d} \in[-9.2 ;-4.6] \times 10^{-4}, \quad a_{s l}^{s} \in[2.0 ; 4.0] \times 10^{-5}$.

\section{ACKNOWLEDGMENTS}

We thank Vladimir Braun, Marco Gersabeck, Thomas Rauh, Alexey Petrov, and Aleksey Rusov for helpful discussions. M. L. P. and C. V. are supported by the UK Science and Technology Facilities Council (STFC) with Grant No. ST/P006744/1.
[1] R. Aaij et al. (LHCb Collaboration), arXiv:1808.08865.

[2] M. Ablikim et al., Chin. Phys. C 44, 040001 (2020).

[3] W. Altmannshofer et al. (Belle-II Collaboration), Prog. Theor. Exp. Phys. 2019, 123C01 (2019); 2020, 029201 (E) (2020).

[4] R. Aaij et al. (LHCb Collaboration), Phys. Rev. Lett. 122, 211803 (2019).

[5] M. Chala, A. Lenz, A. V. Rusov, and J. Scholtz, J. High Energy Phys. 07 (2019) 161.

[6] A. Dery and Y. Nir, J. High Energy Phys. 12 (2019) 104.

[7] A. Khodjamirian and A. A. Petrov, Phys. Lett. B 774, 235 (2017).

[8] H.-N. Li, C.-D. L, and F.-S. Yu, arXiv:1903.10638.

[9] Y. Grossman and S. Schacht, J. High Energy Phys. 07 (2019) 020.

[10] H.-Y. Cheng and C.-W. Chiang, Phys. Rev. D 100, 093002 (2019).

[11] A. Soni, Proc. Sci., LATTICE2019 (2020) 111 [arXiv:2001 .10014].

[12] A. Lenz, in Proceedings, 6th International Workshop on Charm Physics (Charm 2013): Manchester, UK, 2013 (2013).

[13] Y.S. Amhis et al. (HFLAV Collaboration), arXiv:1909 .12524 .

[14] E. Aitala et al. (E791 Collaboration), Phys. Rev. Lett. 77, 2384 (1996).

[15] C. Cawlfield et al. (CLEO Collaboration), Phys. Rev. D 71, 077101 (2005).

[16] B. Aubert et al. (BABAR Collaboration), Phys. Rev. D 76, 014018 (2007).

[17] U. Bitenc et al. (Belle Collaboration), Phys. Rev. D 77, 112003 (2008).

[18] E. Aitala et al. (E791 Collaboration), Phys. Rev. D 57, 13 (1998).

[19] R. Godang et al. (CLEO Collaboration), Phys. Rev. Lett. 84, 5038 (2000).
[20] J. Link et al. (FOCUS Collaboration), Phys. Lett. B 618, 23 (2005).

[21] L. Zhang et al. (Belle Collaboration), Phys. Rev. Lett. 96, 151801 (2006).

[22] B. Aubert et al. (BABAR Collaboration), Phys. Rev. Lett. 98, 211802 (2007).

[23] T. A. Aaltonen et al. (CDF Collaboration), Phys. Rev. Lett. 111, 231802 (2013).

[24] B. Ko et al. (Belle Collaboration), Phys. Rev. Lett. 112, 111801 (2014); 112, 139903(A) (2014).

[25] R. Aaij et al. (LHCb Collaboration), Phys. Rev. D 97, 031101 (2018).

[26] B. Aubert et al. (BABAR Collaboration), Phys. Rev. Lett. 103, 211801 (2009).

[27] R. Aaij et al. (LHCb Collaboration), Phys. Rev. Lett. 116, 241801 (2016).

[28] E. Aitala et al. (E791 Collaboration), Phys. Rev. Lett. 83, 32 (1999).

[29] J. Link et al. (FOCUS Collaboration), Phys. Lett. B 485, 62 (2000).

[30] S. Csorna et al. (CLEO Collaboration), Phys. Rev. D 65, 092001 (2002).

[31] J. Lees et al. (BABAR Collaboration), Phys. Rev. D 87, 012004 (2013).

[32] T. A. Aaltonen et al. (CDF Collaboration), Phys. Rev. D 90, 111103 (2014).

[33] M. Ablikim et al. (BESIII Collaboration), Phys. Lett. B 744, 339 (2015).

[34] R. Aaij et al. (LHCb Collaboration), J. High Energy Phys. 04 (2015) 043.

[35] M. Starič et al. (Belle Collaboration), Phys. Lett. B 753, 412 (2016).

[36] R. Aaij et al. (LHCb Collaboration), Phys. Rev. Lett. 118, 261803 (2017).

[37] R. Aaij et al. (LHCb Collaboration), Phys. Rev. Lett. 122, 011802 (2019). 
[38] B. Aubert et al. (BABAR Collaboration), Phys. Rev. Lett. 100, 061803 (2008).

[39] A. Di Canto (CDF Collaboration), Nuovo Cimento C 036, 26 (2013).

[40] P. del Amo Sanchez et al. (BABAR Collaboration), Phys. Rev. Lett. 105, 081803 (2010).

[41] T. Peng et al. (Belle Collaboration), Phys. Rev. D 89, 091103 (2014).

[42] R. Aaij et al. (LHCb Collaboration), J. High Energy Phys. 04 (2016) 033.

[43] R. Aaij et al. (LHCb Collaboration), Phys. Rev. Lett. 122, 231802 (2019).

[44] A. Zupanc et al. (Belle Collaboration), Phys. Rev. D 80, 052006 (2009).

[45] J. Lees et al. (BABAR Collaboration), Phys. Rev. D 93, 112014 (2016).

[46] D. Asner et al. (CLEO Collaboration), Phys. Rev. D 86, 112001 (2012).

[47] H. N. Nelson, Lepton and photon interactions at high energies, in Proceedings, 19th International Symposium, LP'99, Stanford, USA, 1999 (1999).

[48] A. A. Petrov, eConf C030603, MEC05 (2003).

[49] A. Cerri et al., Report from Working Group 4: Opportunities in Flavour Physics at the HL-LHC and HE-LHC, in Report on the Physics at the HL-LHC, and Perspectives for the HE-LHC (2019), Vol. 7, pp. 867-1158.

[50] V. A. Khoze and M. A. Shifman, Sov. Phys. Usp. 26, 387 (1983).

[51] M. A. Shifman and M. Voloshin, Sov. Phys. JETP 64, 698 (1986).

[52] I. I. Bigi, N. Uraltsev, and A. Vainshtein, Phys. Lett. B 293, 430 (1992); 297, 477(E) (1992).

[53] B. Blok and M. A. Shifman, Nucl. Phys. B399, 441 (1993).

[54] B. Blok and M. A. Shifman, Nucl. Phys. B399, 459 (1993).

[55] J. Chay, H. Georgi, and B. Grinstein, Phys. Lett. B 247, 399 (1990).

[56] M. E. Luke, Phys. Lett. B 252, 447 (1990).

[57] A. Lenz, Lifetimes and heavy quark expansion, in $Q C D$ and Heavy Quarks: In Memoriam Nikolai Uraltsev (2015), Vol. 30, p. 1543005.

[58] M. Kirk, A. Lenz, and T. Rauh, J. High Energy Phys. 12 (2017) 068.

[59] A. Lenz and G. Tetlalmatzi-Xolocotzi, J. High Energy Phys. 07 (2020) 177.

[60] C. T. Davies, J. Harrison, G. P. Lepage, C. J. Monahan, J. Shigemitsu, and M. Wingate (HPQCD Collaboration), Phys. Rev. Lett. 124, 082001 (2020).

[61] R. Dowdall, C. Davies, R. Horgan, G. Lepage, C. Monahan, J. Shigemitsu, and M. Wingate, Phys. Rev. D 100, 094508 (2019).

[62] D. King, A. Lenz, and T. Rauh, J. High Energy Phys. 05 (2019) 034.

[63] L. Di Luzio, M. Kirk, A. Lenz, and T. Rauh, J. High Energy Phys. 12 (2019) 009.
[64] A. Lenz and T. Rauh, Phys. Rev. D 88, 034004 (2013).

[65] U. Nierste, Heavy quark physics, in Proceedings, Helmholtz. International School, HQP08, Dubna, Russia, 2008 (2009), pp. $1-38$.

[66] T. Jubb, M. Kirk, A. Lenz, and G. Tetlalmatzi-Xolocotzi, Nucl. Phys. B915, 431 (2017).

[67] M. Beneke, G. Buchalla, and I. Dunietz, Phys. Rev. D 54, 4419 (1996); 83, 119902 (2011).

[68] M. Beneke, G. Buchalla, C. Greub, A. Lenz, and U. Nierste, Phys. Lett. B 459, 631 (1999).

[69] A. Dighe, T. Hurth, C. Kim, and T. Yoshikawa, Nucl. Phys. B624, 377 (2002).

[70] M. Beneke, G. Buchalla, A. Lenz, and U. Nierste, Phys. Lett. B 576, 173 (2003).

[71] M. Ciuchini, E. Franco, V. Lubicz, F. Mescia, and C. Tarantino, J. High Energy Phys. 08 (2003) 031.

[72] A. Lenz and U. Nierste, J. High Energy Phys. 06 (2007) 072 .

[73] A. Bazavov et al., Phys. Rev. D 97, 034513 (2018).

[74] M. Tanabashi et al. (Particle Data Group), Phys. Rev. D 98, 030001 (2018).

[75] J. Charles, A. Hocker, H. Lacker, S. Laplace, F. Le Diberder, J. Malcles, J. Ocariz, M. Pivk, and L. Roos (CKMfitter Group), Eur. Phys. J. C 41, 1 (2005).

[76] S. Aoki et al. (Flavour Lattice Averaging Group), Eur. Phys. J. C 80, 113 (2020).

[77] S. L. Glashow, J. Iliopoulos, and L. Maiani, Phys. Rev. D 2 , 1285 (1970).

[78] E. Golowich and A. A. Petrov, Phys. Lett. B 625, 53 (2005).

[79] M. Bobrowski, A. Lenz, J. Riedl, and J. Rohrwild, J. High Energy Phys. 03 (2010) 009.

[80] H. Georgi, Phys. Lett. B 297, 353 (1992).

[81] T. Ohl, G. Ricciardi, and E. H. Simmons, Nucl. Phys. B403, 605 (1993).

[82] I. I. Bigi and N. G. Uraltsev, Nucl. Phys. B592, 92 (2001).

[83] M. Bobrowski, A. Lenz, and T. Rauh, in Proceedings, 5th International Workshop on Charm Physics (Charm 2012): Honolulu, Hawaii, USA, 2012 (2012).

[84] A. F. Falk, Y. Grossman, Z. Ligeti, and A. A. Petrov, Phys. Rev. D 65, 054034 (2002).

[85] H.-Y. Cheng and C.-W. Chiang, Phys. Rev. D 81, 114020 (2010).

[86] H.-Y. Jiang, F.-S. Yu, Q. Qin, H.-n. Li, and C.-D. L, Chin. Phys. C 42, 063101 (2018).

[87] H. Asatrian, A. Hovhannisyan, U. Nierste, and A. Yeghiazaryan, J. High Energy Phys. 10 (2017) 191.

[88] H. M. Asatrian, H. H. Asatryan, A. Hovhannisyan, U. Nierste, S. Tumasyan, and A. Yeghiazaryan, Phys. Rev. D 102, 033007 (2020).

[89] A. L. Kagan and L. Silvestrini, arXiv:2001.07207.

[90] H.-N. Li, H. Umeeda, F. Xu, and F.-S. Yu, Phys. Lett. B 810, 135802 (2020).

[91] A. F. Falk, Y. Grossman, Z. Ligeti, Y. Nir, and A. A. Petrov, Phys. Rev. D 69, 114021 (2004). 